\title{
Function characterization of a glyco-engineered anti-EGFR monoclonal antibody cetuximab in vitro
}

\author{
Chang-hong $\mathrm{YI}^{1}$, Can-ping RUAN ${ }^{2}$, Hao WANG ${ }^{3}$, Xin-yun $\mathrm{XU}^{2}$, Yun-peng $\mathrm{ZHAO}^{1}$, Meng $F A N \mathrm{G}^{1}$, Jun $\mathrm{JI}^{1}$, Xing $\mathrm{GU}^{1}$, \\ Chun-fang $\mathrm{GAO}^{1, *}$ \\ ${ }^{1}$ Department of Laboratory Medicine, Eastern Hepatobiliary Surgery Hospital, Second Military Medical University, Shanghai 200438, \\ China; ${ }^{2}$ Department of General Surgery, Changzheng Hospital, Second Military Medical University, Shanghai 200003, China; \\ ${ }^{3}$ Department of Laboratory Medicine, Changzheng Hospital, Second Military Medical University, Shanghai 200003, China
}

\begin{abstract}
Aim: To evaluate the biochemical features and activities of a glyco-engineered form of the anti-human epidermal growth factor receptor monoclonal antibody (EGFR mAb) cetuximab in vitro.

Methods: The genes encoding the Chinese hamster bisecting glycosylation enzyme (GnTIII) and anti-human EGFR mAb were cloned and coexpressed in CHO DG44 cells. The bisecting-glycosylated recombinant EGFR mAb (bisec-EGFR mAb) produced by these cells was characterized with regard to its glycan profile, antiproliferative activity, Fc receptor binding affinity and cell lysis capability. The content of galactose- $\alpha-1,3$-galactose ( $\alpha$-Gal) in the bisec-EGFR mAb was measured using HPAEC-PAD.

Results: The bisec-EGFR mAb had a higher content of bisecting N-acetylglucosamine residues. Compared to the wild type EGFR $\mathrm{mAb}$, the bisec-EGFR mAb exhibited 3-fold higher cell lysis capability in the antibody-dependent cellular cytotoxicity assay, and 1.36fold higher antiproliferative activity against the human epidermoid carcinoma line A431. Furthermore, the bisec-EGFR mAb had a higher binding affinity for human FcyRla and FcyRllla-158F than the wild type EGFR mAb. Moreover, $\alpha$-Gal, which was responsible for cetuximab-induced hypersensitivity reactions, was not detected in the bisec-EGFR mAb.

Conclusion: The glyco-engineered EGFR mAb with more bisecting modifications and lower $\alpha$-Gal content than the approved therapeutic antibody Erbitux shows improved functionality in vitro, and requires in vivo validations.
\end{abstract}

Keywords: EGFR mAb; Erbitux; glyco-engineering; antibody-dependent cellular cytotoxicity; antiproliferation; Fc receptor binding; galactose- $\alpha$-1,3-galactose

Acta Pharmacologica Sinica (2014) 35: 1439-1446; doi: 10.1038/aps.2014.77; published online 29 Sep 2014

\section{Introduction}

More than 300 monoclonal antibodies are in clinical trials for the treatment of human malignancies and inflammation ${ }^{[1,2]}$. The clinical success of this drug class is demonstrated by the large number of therapeutic antibodies that have been brought to market and the increasing number of therapeutic antibodies in development. However, improving the efficacy of therapeutic antibodies and decreasing their side effects remain challenges ${ }^{[1]}$.

Glycosylation is one of the most important post-translational protein modifications and has essential roles in therapeutic antibody function, including effector function, immunogenicity, half-life in serum and other aspects of bioactivity ${ }^{[3,4]}$. Some glycoforms of human antibodies exhibit stronger therapeutic

\footnotetext{
* To whom correspondence should be addressed.

E-mail gaocf1115@163.com

Received 2014-03-12 Accepted 2014-07-05
}

effects than other glycoforms, and some glycoforms possess undesired properties. For example, de-fucosylated Herceptin is at least 50-fold more active in Fcy receptor IIIa-mediated antibody-dependent cellular cytotoxicity (ADCC) assays than the form with alpha-1,6-linked fucose residues ${ }^{[5]}$. The antiCD20 antibody with a higher content of bisecting acetylglucosamine (GlcNAc) residues has 10-20 times higher ADCC than its wild type counterpart ${ }^{[6]}$.

The therapeutic anti-human epidermal growth factor receptor monoclonal antibody (EGFR mAb) cetuximab (marketed under the name Erbitux) is expressed in the murine cell line Sp2/0 and approved for the treatment of colorectal cancer. This therapeutic antibody can bind EGFR and lead to EGFR autophosphorylation, as well as subsequent activation of signal transduction pathways that are involved in regulating cellular proliferation, differentiation, and survival ${ }^{[7]}$. Furthermore, this therapeutic EGFR mAb exhibits ADCC against cancer cells ${ }^{[8]}$. 
A high prevalence of hypersensitivity reactions to cetuximab have been reported in some areas of the United States ${ }^{[9]}$. In most subjects who had a hypersensitivity reaction, IgE antibodies that recognize the EGFR mAb were present in the circulation before treatment. The IgE antibodies were specific for galactose- $\alpha-1,3$-galactose ( $\alpha$-Gal), which is present on the Fab portion of the EGFR mAb heavy chain. Those patients who have such IgE antibodies before treatment tend to develop a hypersensitivity reaction after intravenous injection of monoclonal antibodies containing a-Gal.

In this study, we first established a stable Chinese hamster ovary (CHO) cell line that can express the wild-type EGFR $\mathrm{mAb}$ and subsequently transfected these cells with cDNA encoding GnTIII (a Golgi-localized enzyme that catalyzes the addition of a bisecting $\mathrm{N}$-acetylglucosamine residue on N-linked oligosaccharide chains). Following coexpression of GnTIII and wild type EGFR mAb in this cell line, we characterized the N-glycan profile of the GnTIII-modified EGFR mAb (bisec-EGFR mAb) using DNA sequencerassisted-fluorophore-assisted capillary electrophoresis (DSAFACE $)^{[10,11]}$ and quantified the a-Gal content. The effects of the EGFR mAb on cell growth, ADCC and FcyR binding capacity were investigated in vitro to assess the functions of the bisecEGFR mAb.

\section{Materials and methods Cell lines}

A431 (ATCC CRL-1555) is a cell line expressing EGFR. Daudi (ATCC CCL-213 ${ }^{\mathrm{TM}}$ ) is a B lymphoblast cell line expressing the inhibitory $\mathrm{F}_{\mathrm{c}}$ receptor (FcyRIIb). HEK293 cells expressing

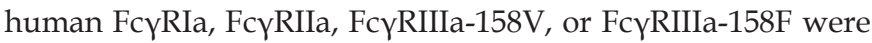
generated as previously described ${ }^{[12]}$.

\section{Expression of wild-type EGFR mAb and bisec-EGFR mAb}

A GnTIII(-) CHO DG44 cell line was used in expression studies of the wild type EGFR mAb and the bisec-EGFR mAb. GnTIII and the heavy chain and light chain of the EGFR mAb (cDNA synthesized by Invitrogen and subcloned into an expression vector in our lab) were coexpressed in CHO DG44 cells using the OptiCHO ${ }^{\mathrm{TM}}$ Antibody Express System (Invitrogen). A cell line that stably expressed the wild type EGFR $\mathrm{mAb}$ was screened with $1 \mu \mathrm{mol} / \mathrm{L}$ MTX and validated by whole cell ELISA (A431) ${ }^{[6]}$. The synthesized Chinese hamster GnTIII gene (NM_001244074.1) was cloned into the pCDNA 3.1 Hygro(-) vector and the wild type EGFR mAb-expressing cell line was transfected with this vector. The bisec-EGFR $\mathrm{mAb}$ (GnTIII gene stably transfected) cell line was selected on $500 \mu \mathrm{g} / \mathrm{mL}$ hygromycin.

Glycosylation analysis of wild type EGFR mAb, bisec-EGFR mAb and the corresponding Fab and Fc fragments

The wild type EGFR mAb and bisec-EGFR mAb from cell supernatants were captured with Protein G-agarose and protein A-agarose, respectively. The corresponding Fc and Fab fragments were isolated using immobilized papain (Thermo Scientific, USA) following the manufacturer's instructions.
The digested supernatant was then loaded onto a Protein A column. The Fab fragments were collected as the flowthrough fraction. The bound Fc fragments were eluted with $0.01 \mathrm{~mol} / \mathrm{L}$ glycine, $\mathrm{pH}$ 3.0. N-glycan profiling of the wild type EGFR mAb, and bisec-EGFR $\mathrm{mAb}$, and the corresponding $\mathrm{Fab}$ and $\mathrm{Fc}$ fragments was conducted by DSA-FACE.

ADCC activity assay of wild type EGFR mAb and bisec-EGFR mAb Peripheral blood mononuclear cells (PBMCs) were separated from heparinized fresh healthy human blood by standard centrifugation procedures using Ficoll/Hypaque (Sigma). The PBMCs used as effector cells were activated in RPMI with $10 \% \mathrm{FBS}$ and $10 \mathrm{U} / \mathrm{mL}$ interleukin-2 (Roche) overnight. The ADCC activity assay was performed according to the manufacturer's instructions (CytoTox $96^{\circledR}$ Non-Radioactive cytotoxicity Assay, Promega, USA). Briefly, A431 cells were grown to the $\log$ phase and resuspended at $4 \times 10^{5}$ cells $/ \mathrm{mL}$ after washing in assay medium (DMEM). The target cells (A431) were added at $50 \mu \mathrm{L} /$ well into a 96-well flat-bottomed cell culture plate. Antibodies were serially diluted in assay medium and then added at $50 \mu \mathrm{L} /$ well in triplicate well in the plates. The plates were incubated at room temperature for $10 \mathrm{~min}$ prior to the addition of $100 \mu \mathrm{L}$ of serially diluted effector cells $(\mathrm{PBMCs})^{[6]}$. The cell mixtures with antibodies were incubated at $37^{\circ} \mathrm{C}$ for $4 \mathrm{~h}$ in a humidified $\mathrm{CO}_{2}$ incubator. One hundred microliters of supernatant was removed from each well and analyzed by measuring lactate dehydrogenase (LDH) activity released from damaged target cells using a CytoTox $96^{\circledR}$ NonRadioactive Cytotoxicity Assay (Promega, USA). The effector and/or target cells were also included as controls. Specific lysis was calculated relative to a total lysis control generated by incubating the target cells with $100 \mu \mathrm{L}$ of $2 \%$ Triton X-100.

\section{Antiproliferative effects of wild type EGFR mAb and bisec-EGFR mAb}

The A431 cell line was employed to test the Fab bindingmediated antiproliferative activity of the antibodies. In brief, A431 cells were incubated with the wild type EGFR mAb and bisec-EGFR mAb diluted in FBS-free medium for $72 \mathrm{~h}$ at $37^{\circ} \mathrm{C}$ with $5 \% \mathrm{CO}_{2}$. After MTS solution (G5340, Promega) was added, the cells were incubated for another $3 \mathrm{~h}$. Colorimetric evaluation was performed at $492 \mathrm{~nm}$ using a spectrophotometer. The inhibition of proliferation is reported as the $\mathrm{IC}_{50}$ induced by the wild type EGFR mAb or bisec-EGFR mAb in comparison with that induced by a positive control (Erbitux).

FcR binding affinity of wild type EGFR mAb and bisec-EGFR mAb HEK293 cells expressing human FcyRIa, FcyRIIa, FcyRIIIa$158 \mathrm{~V}$, or FcyRIIIa-158F $\left(1 \times 10^{6}\right.$ cells $)$ were incubated with the wild type EGFR mAb, bisec-EGFR mAb or Erbitux $(10 \mu \mathrm{g} / \mathrm{mL})$ or $1 \%$ BSA in PBS at $4{ }^{\circ} \mathrm{C}$ for $1 \mathrm{~h}$ and then washed and stained with FITC-labeled anti-human IgG (Sigma, USA). Cells were analyzed using light-scatter parameters on a MACS QUANT flow cytometer (Miltenyi Biotec, Germany). Blank controls were used, setting the cutoff at no more than $0.5 \%$ cells binding with FITC labeled anti-human IgG. 


\section{$\alpha$-Gal quantification of Erbitux and bisec-EGFR mAb}

The standards for the calibration curve were created through serial dilution of a $100 \mathrm{mmol} / \mathrm{L} D$-galactose stock solution (Sigma). Each sample was mixed with $2 \mu \mathrm{L}$ of $(1-3,4,6)$-galactosidase (Prozyme) and diluted to a final concentration of $1 \mathrm{mg} / \mathrm{mL}$ in the reaction buffer and incubated overnight at $37^{\circ} \mathrm{C}$. High-performance anion exchange chromatographic separation of the analyte was performed using an ICS-3000 chromatography system (HPAEC, Dionex Corp). The solution was diluted $2 \times$ to an antibody concentration of $0.5 \mathrm{mg} / \mathrm{mL}$ and detected using a pulsed amperometric detector (PAD) after elution through a CarboPac PA1 column.

\section{Statistical analysis}

All statistical analyses were performed with SPSS 16.0 for Windows software (SPSS, Chicago, IL, USA). Quantitative variables were compared using Student's $t$ test and ANOVA. All reported $P$-values were two-tailed, and $P$ values less than 0.05 were considered to be statistically significant.

\section{Results}

\section{(Bisec-)EGFR mAb expression and N-Glycan analysis}

The EGFR mAb was captured from the cell supernatant by using Protein A. Whole cell ELISA showed that the recombinant wild-type EGFR mAb exhibited dose-dependent binding to A431 cells, comparable to Erbitux (Figure 1).

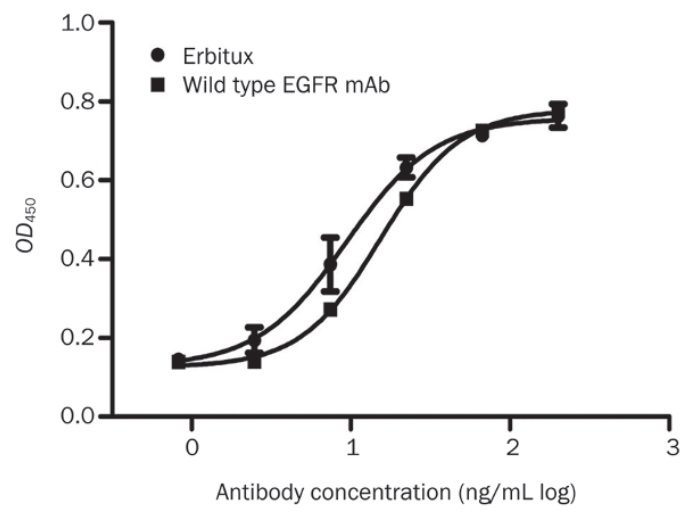

Figure 1. Whole cell ELISA. The wild type EGFR mAb exhibited dosedependent binding to A431 cells, comparable to Erbitux.

$\mathrm{N}$-glycan analysis revealed 7 dominant N-glycan structures (peaks) in the N-glycan profile, with only peak 2 and peak 7 containing bisecting GlcNAc residues ${ }^{[10,11]}$. Thus, the glycanmodified EGFR mAb with elevated abundances of peak 2 and peak 7 (bisec-EGFR mAb) was selected for further study. Typical N-glycan profiles of the wild type EGFR mAb and bisec-EGFR $\mathrm{mAb}$ are shown in Figure 2A-2B. For the wild type EGFR mAb, no bisecting GlcNAc glycoform was found in either the corresponding Fab or Fc fragments (Figure 2A). As expected, the bisecting GlcNAc content (peak 2 and peak 7) was elevated dramatically in both the Fab and Fc fragments of
bisec-EGFR mAb (Figure 2B) compared to the wide type EGFR $\mathrm{mAb}$. Differences in N-glycan abundances between wild type EGFR $\mathrm{mAb}$ and bisec-EGFR $\mathrm{mAb}$ are shown in Figure 2C-2E. Notably, only galactosylated bisecting glycan (peak 2, 24\%) was detected in Fc fragment of bisec-EGFR mAb (Figure 2E), while both agalactosylated and bigalactosylated bisecting GlcNAc (peak 2, 21\%; peak 7, 21\%) glycoforms were detected in the intact antibody (Figure 2C) and Fab fragment (Figure 2D).

\section{Bisec-EGFR mAb had increased ADCC}

Both the wild type EGFR mAb and bisec-EGFR mAb effectively induced A431 cell lysis at effector:target cell (E:T) ratios of 5:1 and higher (Figure 3). The bisec-EGFR mAb showed a stronger lysis effect even at lower E:T ratios $(P<0.05)$. At an $\mathrm{E}: \mathrm{T}$ ratio of 40:1, the bisec-EGFR $\mathrm{mAb}$ yielded effective specific lysis with an approximately three-fold higher effect than that of the wild type EGFR mAb $(89 \%$ vs $30 \%, P<0.001)$. The results indicated that the EGFR $\mathrm{mAb}$ that contained a larger amount of bisecting GlcNAc had a higher ADCC activity.

\section{Bisec-EGFR mAb had higher antiproliferative efficacy than wild type EGFR mAb}

The antiproliferative activity of the EGFR $\mathrm{mAb}$ is mediated by the binding of the Fab fragment to EGFR. As indicated in Figure 4, both the wild type EGFR $\mathrm{mAb}$ and bisec-EGFR $\mathrm{mAb}$ showed significant inhibition of tumor cell growth. The 50\% inhibitory concentration $\left(\mathrm{IC}_{50}\right)$ values of Erbitux, the wild type EGFR $\mathrm{mAb}$ and the bisec-EGFR $\mathrm{mAb}$ were 0.162 (95\% CI: 0.143-0.184), 0.178 (95\% CI: 0.159-0.201) and 0.119 (95\% CI: 0.095-0.150) $\mathrm{mg} / \mathrm{mL}$, respectively. Thus, the bisec-EGFR mAb was approximately 1.36-fold more potent than Erbitux, and the potency of the wild type EGFR mAb was comparable to that of the positive control (Erbitux).

Bisec-EGFR mAb had improved binding affinity for Fcy receptors The binding activities of the wild type EGFR $\mathrm{mAb}$, bisecEGFR mAb, and Erbitux to $\mathrm{Fcy}_{\mathrm{c}}$ receptors (FcyRs, both inhibitory and activating $\mathrm{F}_{\mathrm{c}} \mathrm{R}$ Rs) were tested by flow cytometry. All of the EGFR mAbs bound to human FcyRs with the following rank order of affinity: Fc $\mathrm{RIa}>\mathrm{Fc}$ RIIIa-158V $>$ Fc $\mathrm{RIII}$ $158 \mathrm{~F}>\mathrm{F}_{\mathrm{c} \gamma \mathrm{RIIa}}>\mathrm{F}_{\mathrm{c} \gamma \mathrm{RIIb}}$ (Figure 5). Compared to the wild type EGFR mAb, the bisec-EGFR mAb showed a higher binding affinity for human FcyRIa $(P=0.001$, Fig ure 5A) and FcyRIIIa$158 \mathrm{~F}(P=0.013$, Figure 5B). The binding affinities for $158 \mathrm{~V}$ (Figure 5C), FcyRIIa (Figure 5D), and FcyRIIb (Figure 5E) of bisecEGFR $\mathrm{mAb}$ and wild-type EGFR mAb were similar.

\section{$\alpha$-Gal quantification of Erbitux and bisec-EGFR mAb}

The digested Erbitux and bisec-EGFR mAb were analyzed in triplicate, and the results were measured against a calibration curve (Figure 6). The a-Gal peak was observed at a retention time of approximately $12 \mathrm{~min}$ after injection of the digested Erbitux (Figure 7A). The molar ratio of a-Gal per Erbitux molecule was determined to be 2.36 $\pm 0.09: 1$. For the bisec-EGFR $\mathrm{mAb}$, no detectable peak was observed at a retention time of 
A
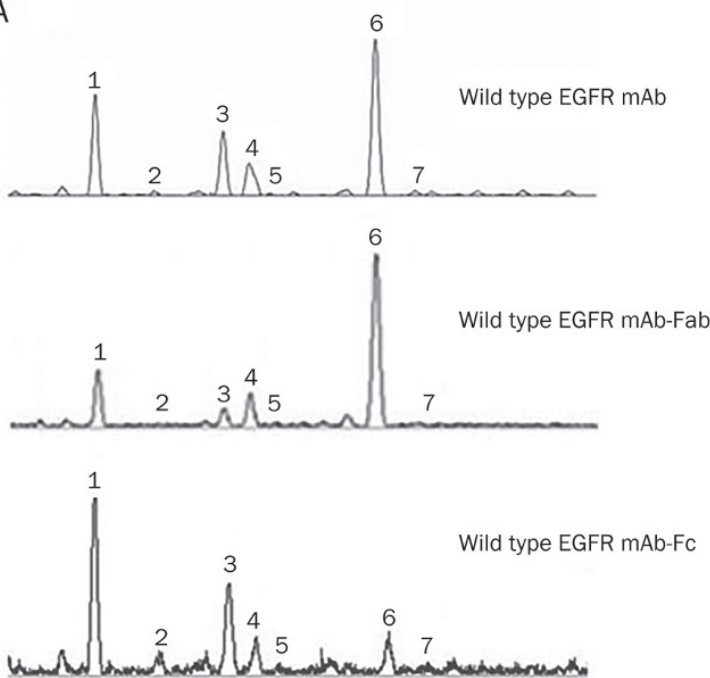

B
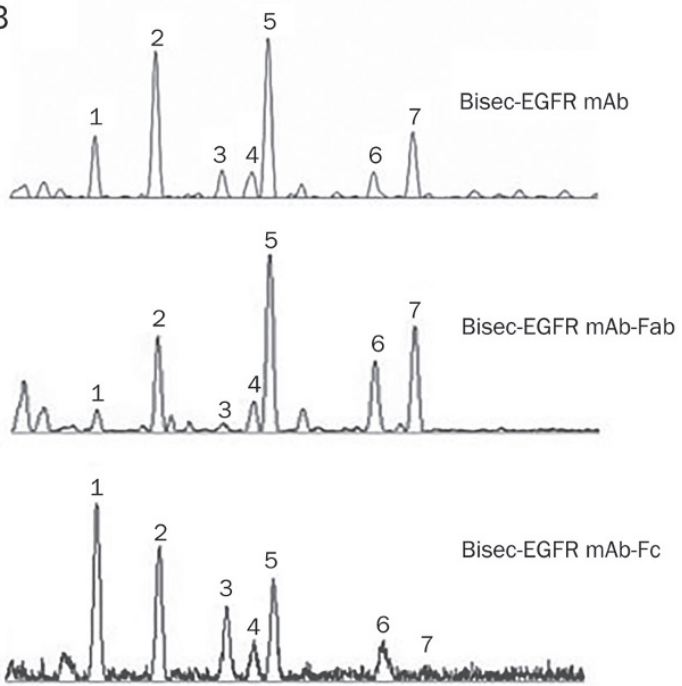

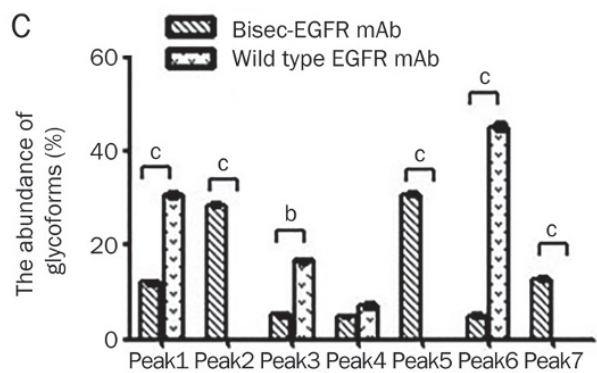

D

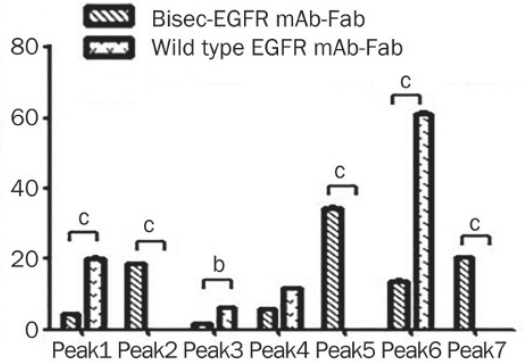

E
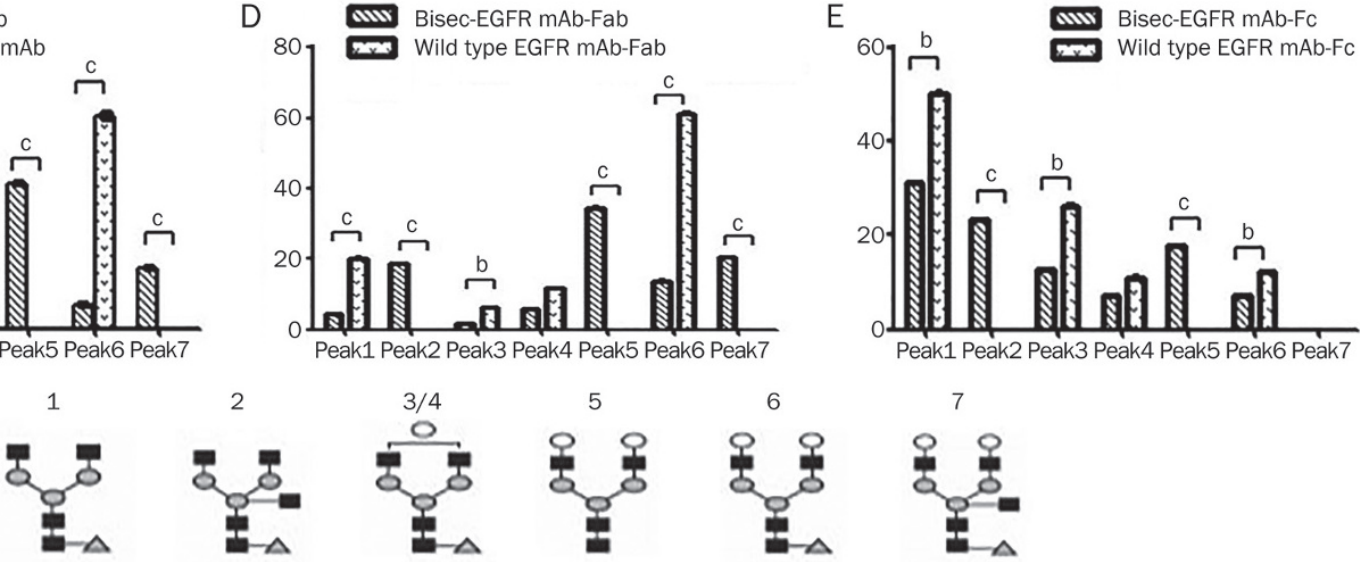

Figure 2. Desialylated N-glycan profiling of wild type EGFR mAb and bisec-EGFR mAb. For the wild type EGFR mAb (A), the bisecting GlcNAc glycoforms: an agalacto core- $\alpha-1,6$-fucosylated bisecting biantennary glycan (NGA2FB, peak 2 ) and a bigalacto core- $\alpha-1,6$-fucosylated bisecting biantennary glycan (NA2FB, peak 7) were not found. However, the bisected GlcNAc content was elevated dramatically in both the Fab and Fc fragments of the bisec-EGFR $\mathrm{mAb}$ (B). Differences in N-glycan abundance between the wild type EGFR mAb and bisec-EGFR mAb are shown in $\mathrm{C}-\mathrm{E}\left({ }^{\mathrm{b}} \mathrm{P}<0.05\right.$, $\left.{ }^{\mathrm{c}} \mathrm{P}<0.01\right)$. Notably, only agalactosylated bisecting GIcNAc (peak 2, E) was detected for the Fc of bisec-EGFR mAb, whereas both agalactosylated and bigalactosylated GIcNAc (peak 2 and peak 7) were detected in the consensus intact antibody (C) and Fab fragment (D).

approximately $12 \mathrm{~min}$ after injection (Figure 7B), and a similar result was found for the wild type EGFR mAb (data not shown).

The retention time of the a-Gal peak variant, at approximately $12 \mathrm{~min}$, is highly sensitive to small changes in the sodium hydroxide concentration and the amount of dissolved carbon dioxide from the air, but the area of the peak is relatively consistent between different runs.

\section{Discussion}

The effector functions elicited by therapeutic antibodies strongly depend on the carbohydrate moiety linked to the antibody protein. Therefore, several approaches have been developed to rationally manipulate these glycans and improve the biological functions of antibodies ${ }^{[13]}$.
GnT-III is a key enzyme in N-glycan biosynthesis and catalyzes the transfer of GlcNAc from UDP-GlcNAc, a glycosyl donor, to a core $\beta$-mannose residue in N-linked oligosaccharides via a $\beta 1 \rightarrow 4$ linkage, resulting in the formation of a bisected sugar chain ${ }^{[14]}$. The GlcNAc residue added is referred to as a bisecting GlcNAc. The addition of this unique structure inhibits the action of other $\mathrm{N}$-acetylglucosaminyl transferases, such as GnT-IV and GnT-V, both of which are involved in the formation of multi-antennary sugar chains ${ }^{[15]}$. Thus, GnT-III is a critical enzyme that has been used to improve the function of therapeutic antibodies.

Increasing evidence shows that $\mathrm{N}$-linked oligosaccharides can affect the solubilities, clearance rates and effector functions of antibody molecules ${ }^{[16]}$. GnT-III is an ideal enzyme for manipulating the N-glycosylation of expressed proteins, as it 


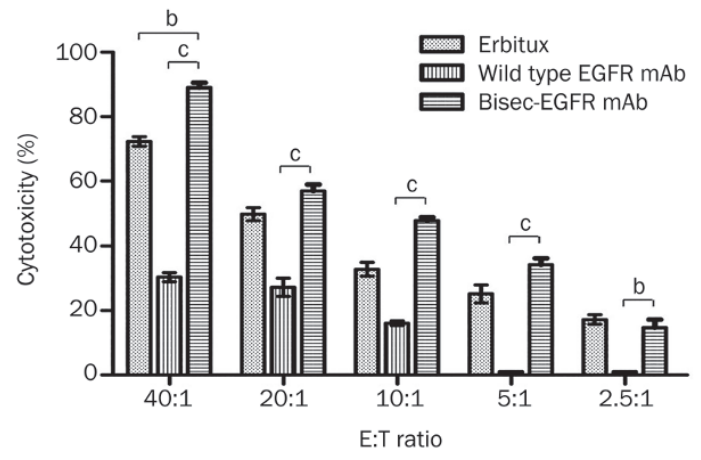

Figure 3. Comparisons of wild type EGFR mAb and bisec-EGFR mAb in ADCC. ADCC was evaluated in EGFR-positive A431 cells at different effector:target cell $(E: T)$ ratios. Compared to the wild type EGFR mAb, the bisec-EGFR mAb resulted in comparable effective specific lysis at a lower E:T ratio $(P<0.05)$. At the same E:T ratio $(40: 1)$, the bisec-EGFR $\mathrm{mAb}$ resulted in effective specific lysis, with approximately three-fold greater lysis than that mediated by the wild type EGFR mAb (88\% vs 30\%, $P<0.001)$. ${ }^{\mathrm{b}} P<0.05,{ }^{\mathrm{C}} P<0.01$.

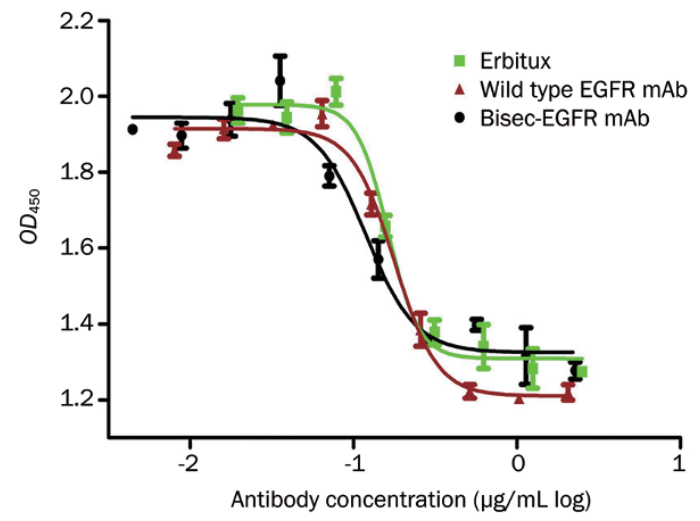

Fab of antibody $\mathrm{IC}_{50}(\mu \mathrm{g} / \mathrm{mL})$ Potency compared to Erbitux (\%)

\begin{tabular}{lrr}
\hline Erbitux & 0.162 & 100 \\
Wild type EGFR mAb & 0.178 & 91 \\
Bisec-EGFR mAb & 0.119 & 136 \\
\hline
\end{tabular}

Figure 4. Antiproliferative efficacies of wild type EGFR mAb and bisecEGFR mAb. Both the wild type EGFR mAb and bisec-EGFR mAb showed significant inhibition of tumor cell growth. The potency of the wild type EGFR mAb was comparable to that of Erbitux. The bisec-EGFR mAb exhibited a potency that was approximately 1.36-fold of that of Erbitux.

exerts a large degree of control over the glycosylation process by blocking the action of 1,6-FucT, ManII, and GnT-II ${ }^{[17,18]}$.

Previous studies have shown that over-expression of GnTIII in CHO DG44 cells leads to ADCC enhancement of an antineuroblastoma $\operatorname{IgG} 1^{[18]}$, an anti-CD20 $\operatorname{IgG1} 1^{[19]}$, and an antihuman interleukin 5 receptor $\operatorname{IgG} 1^{[20]}$. To further identify the roles of bisecting GlcNAc in EGFR mAb function, we over- expressed GnTIII in an EGFR mAb producing cell line (CHO DG44) that lacks endogenous GnTIII expression ${ }^{[21]}$, and we confirmed that an antibody with bisecting GlcNAc (the bisecEGFR $\mathrm{mAb}$ ) enhanced ADCC compared to an antibody without bisecting GlcNAc (the wild type EGFR mAb).

However, all of the previous studies have only focused on the glycosylation of intact IgG. Considering the different oligosaccharide profiles of IgG Fab and Fc fragments revealed in some case-control studies ${ }^{[22,23]}$, we also analyzed the N-glycan profiles of antibody Fab and Fc fragments. Both the Fab and Fc fragments of the bisec-EGFR mAb showed an increased bisecting GlcNAc content compared to the wild type EGFR $\mathrm{mAb}$, but the Fab and Fc fragments had different $\mathrm{N}$-glycan profiles from each other. This finding demonstrated site specificity for the elongation of the oligosaccharide chains on the glycoprotein ${ }^{[2]}$ and may explain why the antibodies with a higher bisecting GlcNAc content had higher ADCC activity. First, the enhanced effect may be due to an increased Fc affinity for $\mathrm{F}_{\mathrm{C} Y}$ receptors ${ }^{[24]}$. Second, the improved ADCC activity may also result from a better specific binding of Fab to antigen-positive cells, which initiates the process of ADCC and target cell lysis ${ }^{[25]}$.

In the Fab-mediated cell antiproliferation assay, we found that the potency of the bisec-EGFR mAb was $50 \%$ higher than that of the wild type EGFR mAb. Meanwhile, a comparative study on $\mathrm{Fc}_{\mathrm{c}}$ receptor binding activity revealed that the bisecEGFR $m A b$ had a higher binding affinity for activated human Fcy receptors (mainly FcRIa and 158F-FcRIIIa) than did the wild type EGFR mAb. Taken together, these data suggest that the bisecting GlcNAc modified antibody has higher affinities for both activated FCR and EGFR-expressing cells.

Most of the recently marketed therapeutic antibodies are manufactured in $\mathrm{CHO}$ cells, in part because of the ability of these cells to produce proteins with desirable properties, including 'human-like' glycosylation profiles. Specific glycan structures may adversely affect an antibody's safety profile. For example, the terminal galactose- $\alpha-1,3$-galactose ( $a-G a l$ ) antigen can react with circulating anti a-Gal antibodies, which are present in most individuals, and induce an anaphylactic response ${ }^{[26]}$. It is now understood that murine cell lines, such as NS0 or SP2/0, contain the necessary biosynthetic machinery to produce proteins containing a-Gal epitopes ${ }^{[27-29]}$. However, it is generally accepted that $\mathrm{CHO}$ cells lack the biosynthetic machinery to synthesize glycoproteins with a-Gal antigens ${ }^{[30]}$. Some reports have revealed that $\mathrm{CHO}$ cells do not produce a-1,3-galactosyltransferase ${ }^{[31]}$ and have a pattern of glycosylation that differs from that of the Erbitux host cell line Sp2/0. However, data from other groups have identified the presence of the $\mathrm{CHO}$ ortholog of $\mathrm{N}$-acetyllactosaminide 3-a-galactosy ltransferase- $1^{[32]}$, which is responsible for the synthesis of the a-Gal epitope. These groups also found that the amounts of terminal a-Gal in $\mathrm{CHO}$ clones ranged from 0 to 404 picomol per mg of protein; therefore, selection of a cell line without a-Gal expression is important for $\mathrm{CHO}$-derived therapeutic antibody production.

In this study, we developed and quantified the concentra- 

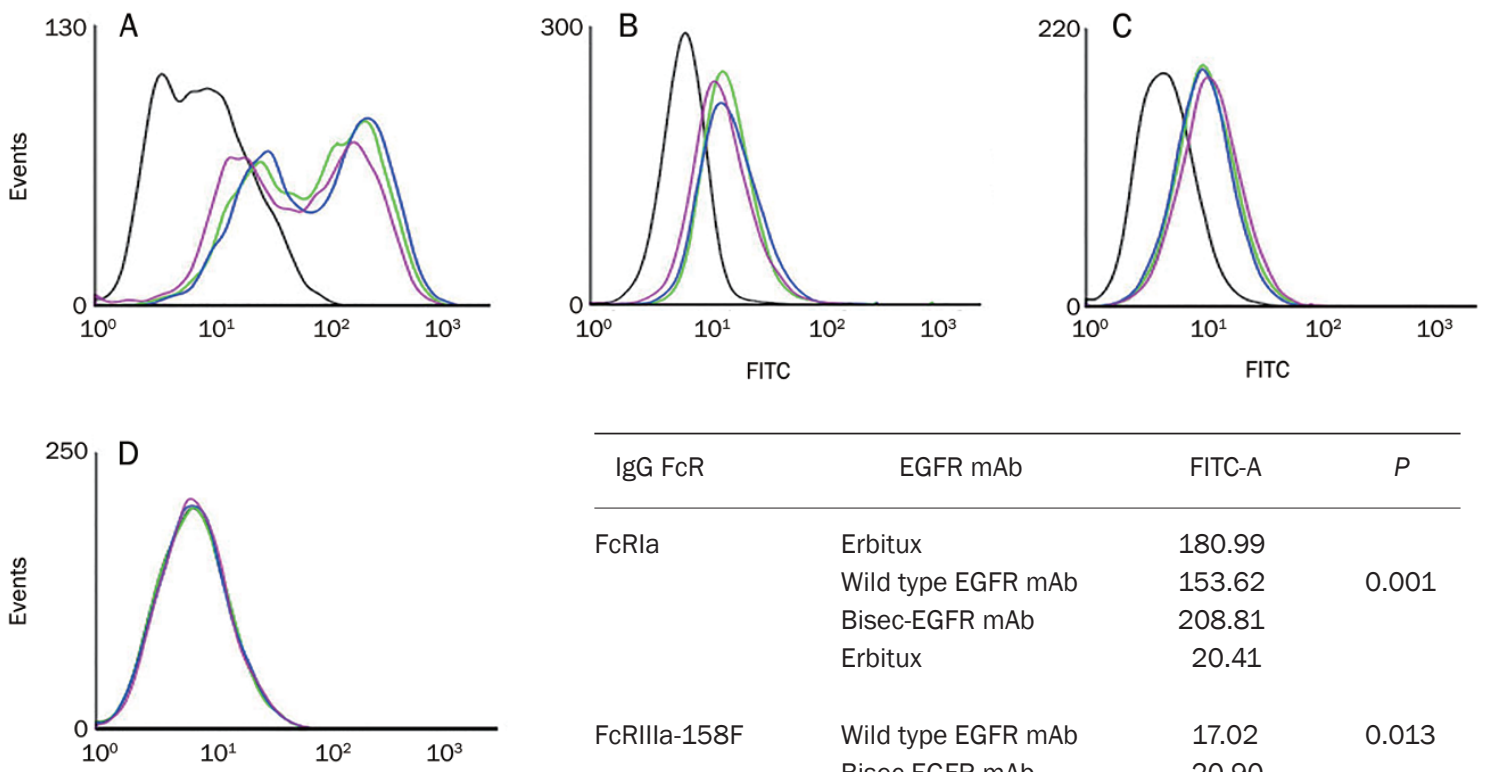

\begin{tabular}{llrl}
\hline \multicolumn{1}{c}{ IgG FcR } & \multicolumn{1}{c}{ EGFR mAb } & FITC-A & $P$ \\
\hline FcRla & Erbitux & 180.99 & \\
& Wild type EGFR mAb & 153.62 & 0.001 \\
& Bisec-EGFR mAb & 208.81 & \\
& Erbitux & 20.41 & \\
FcRIIIa-158F & Wild type EGFR mAb & 17.02 & 0.013 \\
& Bisec-EGFR mAb & 20.90 & \\
& Erbitux & 14.59 & \\
FcRIIIa-158V & Wild type EGFR mAb & 16.02 & 0.108 \\
& Bisec-EGFR mAb & 13.38 & \\
& Erbitux & 9.47 & \\
FcRIIa & Wild type EGFR mAb & 9.46 & 0.163 \\
& Bisec-EGFR mAb & 9.79 & \\
& Erbitux & 10.12 & \\
FcRIIb & Wild type EGFR mAb & 11.16 & 0.077 \\
& Bisec-EGFR mAb & 12.36 & \\
\hline
\end{tabular}

Figure 5. Binding affinities of wild type EGFR mAb and bisec-EGFR mAb to FcyRa-positive and FcyRllb-positive cells. The binding affinity of the wild type EGFR mAb (purple line), bisec-EGFR mAb (blue line), and Erbitux (green line) for FcyRs was tested by flow cytometry. The yellow line represents the blank control. The EGFR mAbs bound to FcyRs with the following rank order: FcyRla $>$ FcyRIIla-158V >FcyRIIla-158F>FcyRlla $>$ FcyRIlb. Compared to the wild type EGFR mAb, the bisec-EGFR mAb showed significantly enhanced binding affinity for FcyRla (A, $P=0.001)$ and FcyRIIla-158F (B, $P=0.013)$. The binding affinities for human FcyRIIla-158V (C), FcyRIla (D), and FcyRIlb (E) were comparable between the bisec-EGFR mAb and the wild type EGFR mAb.

tion of $a-G a l$ in the bisec-EGFR mAb using HPAEC-PAD (high-performance anion exchange chromatography-pulsed amperometric detection). Consistent with a previous report ${ }^{[33]}$, we found that Erbitux contains an a-Gal epitope (2.36 \pm 0.09 $\mu$ mol per $\mu$ mol Erbitux), whereas the bisec-EGFR mAb we investigated did not show an obvious a-Gal peak. Although the specific levels of a-Gal required to trigger anaphylactic reactions are not yet clear, the fact that patients have high levels of circulating anti-a-Gal antibodies ${ }^{[9]}$ suggests that controlling the levels of the a-Gal epitope during EGFR mAb development may have a positive impact on drug safety. More evidence for avoiding the hypersensitivity reaction to a-Gal epitope could be provided by clinical trials.

In conclusion, we modified the glyco-structure of the EGFR $\mathrm{mAb}$ by increasing the bisecting glycan abundance. This bisec-EGFR mAb showed higher ADCC and lower a-Gal lev- els than the approved therapeutic antibody (Erbitux). Additionally, the glycan-modified antibody showed higher binding to FcyRIa and FcyRIIIa-158F. These results indicate that the glyco-engineering of anti-EGFR antibodies could optimize their function and minimize their side effects (such as hypersensitivity). Validation in vivo will be required to confirm the improved efficacy of the glyco-engineered antibody. This research might provide a new, alternative, strategy for the production of engineered therapeutic monoclonal antibodies.

\section{Acknowledgements}

This work was supported by National Natural Science Foundation of China (№ 81301877 and 81271925); State Key Project on Infectious Diseases of China (№ 2012ZX10002-016); Science and Technology Commission of Shanghai Municipality (№ 11JC1416400). 


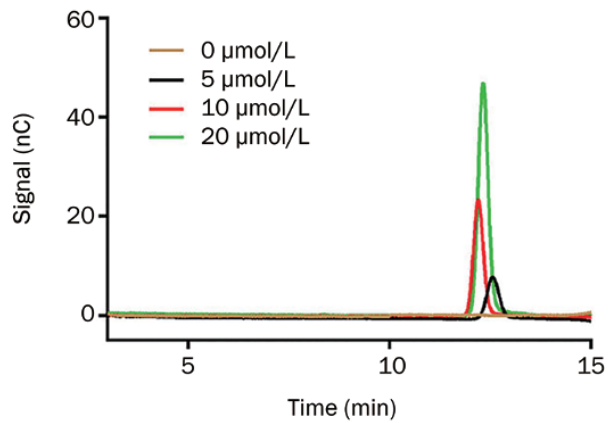

Figure 6. $\alpha$-Galactose standard curve building. By high-performance anion exchange chromatography-pulsed amperometric detection (HPAECPAD), an $\alpha$-Galactose standard curve was created by the serial dilution of a $100 \mathrm{mmol} / \mathrm{L}$ D-galactose stock solution (Sigma). The $\alpha$-Gal peak was observed at a retention time of approximately $12 \mathrm{~min}$ after the injection of D-galactose solution.
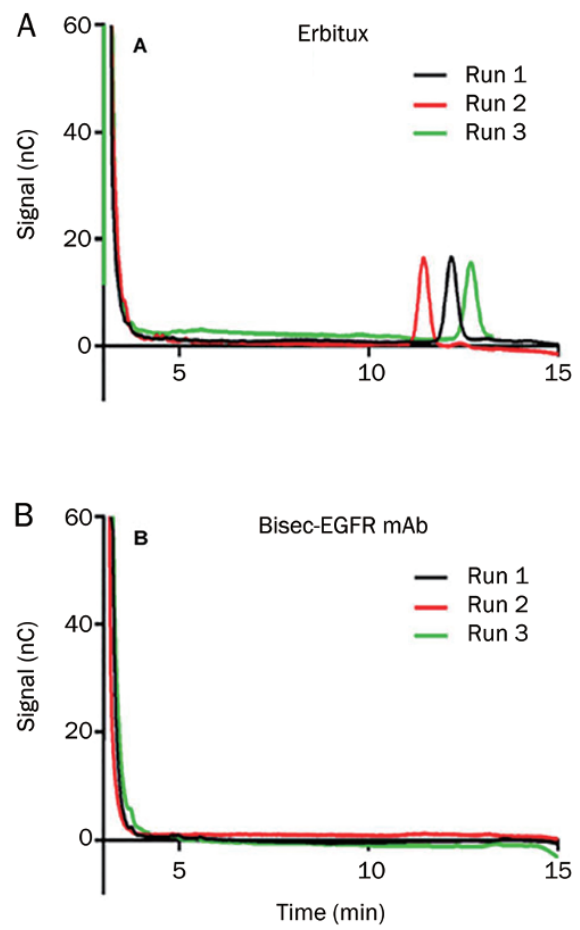

Figure 7. $\alpha$-Galactose analysis of Erbitux and bisec-EGFR mAb. Digested Erbitux and bisec-EGFR mAb were analyzed in triplicate, and the results were measured against the standard curve. The $\alpha$-Gal peak was observed in digested Erbitux (A). For the bisec-EGFR mAb, no detectable $\alpha$-Gal peak was observed (B).

\section{Author contribution}

Chang-hong YI, Can-ping RUAN, and Chun-fang GAO designed the research; Chang-hong YI, Xin-yun XU, Yunpeng ZHAO, and Meng FANG performed research; Jun JI, Hao WANG, and Xing GU analyzed data; Chang-hong YI and Chun-fang GAO wrote the paper.

\section{References}

1 Hansel TT, Kropshofer H, Singer T, Mitchell JA, George AJ. The safety and side effects of monoclonal antibodies. Nat Rev Drug Discov 2010; 9: 325-38.

2 Zhu Z. Targeted cancer therapies based on antibodies directed against epidermal growth factor receptor: status and perspectives. Acta Pharmacol Sin 2007; 28: 1476-93.

3 Jefferis R. Glycosylation of recombinant antibody therapeutics. Biotechnol Prog 2005; 21: 11-6.

4 Li J, Zhu Z. Research and development of next generation of antibodybased therapeutics. Acta Pharmacol Sin 2010; 31: 1198-207.

5 Shields RL, Lai J, Keck R, O'Connell LY, Hong K, Meng YG, et al. Lack of fucose on human IgG1 N-Linked oligosaccharide improves binding to human FcyRIII and antibody-dependent cellular toxicity. J Biol Chem 2002; 277: 26733-40.

6 Davies J, Jiang L, Pan LZ, LaBarre MJ, Anderson D, Reff M. Expression of $\mathrm{GnTIII}$ in a recombinant anti-CD20 $\mathrm{CHO}$ production cell line: expression of antibodies with altered glycoforms leads to an increase in ADCC through higher affinity for FcgRIII. Biotechnol Bioeng 2001; 74: 288-94.

7 Matsuo T, Nishizuka SS, Ishida K, Iwaya T, Ikeda M, Wakabayashi G. Analysis of the anti-tumor effect of cetuximab using protein kinetics and mouse xenograft models. BMC Res Notes 2011; 4: 140-7.

8 Kurai J, Chikumi H, Hashimoto K, Yamaguchi K, Yamasaki A, Sako T, et al. Antibody-dependent cellular cytotoxicity mediated by cetuximab against lung cancer cell lines. Clin Cancer Res 2007; 13: 1552-61.

9 Chung $\mathrm{CH}$, Mirakhur B, Chan E, Le QT, Berlin J, Morse M, et al. Cetuximab-Induced Anaphylaxis and IgE Specific for Galactose- $\alpha-1,3-$ Galactose. N Engl J Med 2008; 358: 1109-17.

10 Callewaert N, Van Vlierberghe H, Van Hecke A, Laroy W, Delanghe $\mathrm{J}$, Contreras R. Noninvasive diagnosis of liver cirrhosis using DNAsequencer based total serum protein glycomics. Nat Med 2004; 10 : 429-34.

11 Liu XE, Desmyter L, Gao CF, Laroy W, Dewaele S, Vanhooren V, et al. $\mathrm{N}$-glycomic changes in hepatocellular carcinoma patients with liver cirrhosis induced by hepatitis B virus. Hepatology 2007; 46: 142635.

12 Arora T, Padaki R, Liu L, Hamburger AE, Ellison AR, Stevens SR, et al. Differences in binding and effector functions between classes of TNF antagonists. Cytokine 2009; 45: 124-31.

13 Ferrara C, Brünker P, Suter T, Moser S, Püntener U, Umaña P. Modulation of therapeutic antibody effector functions by glycosylation engineering: influence of Golgi enzyme localization domain and coexpression of heterologous beta1,4-N-acetylglucosaminyltransferase III and Golgi alpha-mannosidase II. Biotechnol Bioeng 2006; 93: 851-61.

14 Narasimhan S. Control of glycoprotein synthesis. UDP-GIcNAc: glycopeptide beta 4-N-acetylglucosaminyl transferase III, an enzyme in hen oviduct which adds GIcNAc in beta 1,4 linkage to the beta-linked mannose of the trimannosyl core of $\mathrm{N}$-glycosyl oligosaccharides. J Biol Chem1982; 257: 10235-42.

15 Taniguchi N, Miyoshi E, Ko JH, Ikeda Y, Thara Y. Implication of $\mathrm{N}$-ace tylglucosaminyltransferases III and V in cancer: gene regulation and signaling mechanism. Biochim Biophys Acta 1999; 1455: 287-300.

16 Arnold JN, Wormald MR, Sim RB, Rudd PM, Dwek RA. The impact of glycosylation on the biological function and structure of human immunoglobulins. Annu Rev Immunol 2007; 25: 21-50.

17 Schachter $\mathrm{H}$. Biosynthetic controls that determine the branching and microheterogeneity of protein-bound oligosaccharides. Biochem Cell Biol 1986; 64: 163-81. 
18 Umaña P, Jean-Mairet J, Moudry R, Amstutz H, Bailey JE. Engineered glycoforms of an antineuroblastoma IgG1 with optimized antibodydependent cellular cytotoxic activity. Nat Biotechnol 1999; 17: 17680.

19 Umaña P, Jean-Mairet J, Bailey JE. Tetracycline-regulated overexpression of glycosyltransferases in Chinese hamster ovary cells. Biotechnol Bioeng 1999; 65: 542-9.

20 Shinkawa T, Nakamura K, Yamane N, Shoji-Hosaka E, Kanda Y, Sakurada $\mathrm{M}$, et al. The absence of fucose but not the presence of galactose or bisecting N-Acetylglucosamine of human IgG1 complextype oligosaccharides shows the critical role of enhancing antibodydependent cellular cytotoxicity. J Biol Chem 2003; 278: 3466-73.

21 Cambell C, Stanley P. A dominant mutation to ricin resistance in Chinese hamster ovary cells induces UDP-GIcNAc: Glycopeptidebeta4-N-acetylglucosaminyl transferase III activity. J Biol Chem 1984; 10: 13370-8.

22 Holland M, Yagi H, Takahashi N, Kato K, Savage CO, Goodall DM, et al. Differential glycosylation of polyclonal lgG, IgG-Fc and lgG-Fab isolated from the sera of patients with ANCA-associated systemic vasculitis. Biochim Biophys Acta 2006; 1760: 669-77.

23 Youings A, Chang SC, Dwek RA, Scragg IG. Site-specific glycosylation of human immunoglobulin $\mathrm{G}$ is altered in four rheumatoid arthritis patients. Biochem J 1996; 314: 621-30.

24 Nimmerjahn F, Ravetch JV. Antibodies, Fc receptors and cancer. Curr Opin Immunol 2007; 19: 239-45.

25 Abès R, Teillaud JL. Impact of glycosylation on effect or functions of therapeutic lgG. Pharmaceuticals 2010; 3: 146-57.

26 Macher BA, Galili U. The Gal $\alpha 1$, 3Galß1,4GIcNAc-R ( $\alpha$-Gal) epitope: A carbohydrate of unique evolution and clinical relevance. Biochim
Biophys Acta 2008; 1780: 75-88.

27 Larsen RD, Rajan VP, Ruff MM, Kukowska-Latallo J, Cummings RD, Lowe JB. Isolation of a cDNA encoding a murine UDP galactose: beta-D-galactosyl-1,4-N-acetyl-D-glucosaminide alpha-1,3-galactosyltransferase: expression cloning by gene transfer. Proc Natl Acad Sci U S A 1989; 86: 8227-31.

28 Sheeley DM, Merrill BM, Taylor LC. Characterization of Monoclonal Antibody Glycosylation: Comparison of Expression Systems and Identification of Terminal $\alpha$-Linked Galactose. Anal Biochem 1997; 247: 102-10.

29 Chung $\mathrm{CH}$, Mirakhur B, Chan E, Le QT, Berlin J, Morse M, et al. Cetuximab-Induced Anaphylaxis and IgE Specific for Galactose- $\alpha-1,3-$ Galactose. N Engl J Med 2008; 358: 1109-17.

30 Jenkins N, Parekh RB, James DC. Getting the glycosylation right: implications for the biotechnology industry. Nat Biotechnol 1996; 14 : 975-81.

31 Jefferis R. Glycosylation of human IgG antibodies: relevance to therapeutic applications. BioPharm 2002; 14: 19-26. Recent research developments in immunology. Vol 4 (2002); Part II, pp. 769780

32 Bosques CJ, Collins BE, Meador JW 3rd, Sarvaiya H, Murphy JL, Dellorusso G, et al. Chinese hamster ovary cells can produce galactose- $\alpha-1,3$-galactose antigens on proteins. Nat Biotechnol 2010; 28: $1153-6$.

33 Lammerts van Bueren JJ, Rispens T, Verploegen S, van der PalenMerkus T, Stapel S, Workman L, et al. Anti-galactose- $\alpha-1,3$-galactose IgE from allergic patients does not bind $\alpha$-galactosylated glycans on intact therapeutic antibody Fc domains. Nat Biotechnol 2011; 29 : 574-6. 